\title{
Host-Dependent Differences in Replication Strategy of the Sulfolobus Spindle-Shaped Virus Strain SSV9 (a.k.a., SSVK1): Infection Profiles in Hosts of the Family Sulfolobaceae
}

\author{
Ruben Michael Ceballos ${ }^{1,2,3 *}$, Coyne Gareth Drummond ${ }^{4}$, Carson Len Stacy ${ }^{3}$, \\ Elizabeth Padilla-Crespo ${ }^{4}$ and Kenneth Mark Stedman ${ }^{5}$
}

${ }^{1}$ Department of Biological Sciences, The University of Arkansas, Fayetteville, AR, United States, ${ }^{2}$ Arkansas Center for Space and Planetary Sciences, Fayetteville, AR, United States, ${ }^{3}$ Cell and Molecular Biology Program, The University of Arkansas, Fayetteville, AR, United States, ${ }^{4}$ Departmento de Ciencias y Tecnología, Universidad Interamericana de Puerto Rico, Aguadilla, PR, United States, ${ }^{5}$ Department of Biology, Center for Life in Extreme Environments, Portland State University, Portland, OR, United States

OPEN ACCESS

Edited by:

Jesse G. Dillon, California State University, Long Beach, United States

Reviewed by: Hanna M. Oksanen, University of Helsinki, Finland Mart Krupovic Institut Pasteur, France

*Correspondence: Ruben Michael Ceballos ceballos@uark.edu

Specialty section: This article was submitted to

Extreme Microbiology, a section of the journal Frontiers in Microbiology

Received: 24 February 2020 Accepted: 13 May 2020 Published: 14 July 2020

Citation:

Ceballos RM, Drummond CG,

Stacy CL, Padilla-Crespo E and Stedman KM (2020) Host-Dependent Differences in Replication Strategy of the Sulfolobus Spindle-Shaped Virus Strain SSV9 (a.k.a., SSVK1): Infection Profiles in Hosts of the Family Sulfolobaceae.

Front. Microbiol. 11:1218. doi: 10.3389/fmicb.2020.01218
The Sulfolobus Spindle-shaped Virus (SSV) system has become a model for studying thermophilic virus biology, including archaeal host-virus interactions and biogeography. Several factors make the SSV system amenable to studying archaeal genetic mechanisms (e.g., CRISPRs) as well as virus-host interactions in high temperature acidic environments. Previously, we reported that SSVs exhibited differential infectivity on allopatric vs. sympatric hosts. We also noticed a wide host range for virus strain SSV9 (a.k.a., SSVK1). For decades, SSVs have been described as "non-lytic" double-stranded DNA viruses that infect species of the genus Sulfolobus and release virions via budding rather than host lysis. In this study, we show that SSVs infect hosts representing more than one genus of the family Sulfolobaceae in spot-on-lawn "halo" assays and in liquid culture infection assays. Growth curve analyses support the hypothesis that SSV9 virion release causes cell lysis. While SSV9 appears to lyse allopatric hosts, on a single sympatric host, SSV9 exhibits canonical non-lytic viral release historically reported SSVs. Therefore, the nature of SSV9 lytic-like behavior may be driven by allopatric evolution. The SSV9-infected host growth profile does not appear to be driven by multiplicity of infection (MOI). Greater stability of SSV9 vs. other SSVs (i.e., SSV1) in high temperature, low pH environments may contribute to higher transmission rates. However, neither higher transmission rate nor relative virulence in SSV9 infection seems to alter replication profile in susceptible hosts. Although it is known that CRISPR-Cas systems offer protection against viral infection in prokaryotes, CRISPRS are not reported to be a determinant of virus replication strategy. The mechanisms underlying SSV9 lytic-like behavior remain unknown and are the subject of ongoing investigations. These results suggest that genetic elements, potentially resulting from allopatric evolution, mediate distinct virus-host growth profiles of specific SSV-host strain pairings.

Keywords: Sulfolobus spindle-shaped virus, lytic replication, allopatric evolution, sympatric coevolution, non-lytic release, Sulfolobus, fusellovirus, SSV9 


\section{INTRODUCTION}

In virology, reduced model systems are prokaryotic or single-cell eukaryotic viral systems that provide fundamental information regarding virus-host interactions and coevolution independent of the complexity found in macroorganisms with evolved immune systems. Reduced model viral systems have been used extensively to study fundamental properties of virus evolution (Lenski and Levin, 1985; Morgan et al., 2005; Brockhurst et al., 2007). Moreover, it is suggested that studying virus-host interactions in reduced model systems may provide opportunities to understand fundamental processes of virus evolution in host systems of agricultural or medical importance (Brockhurst et al., 2007; Dennehy, 2009). Given the recent view that the evolutionary origin(s) of viruses may be linked to the early evolution of Archaea (Forterre, 2006; Berliner et al., 2018) and the suggestion that the emergence of viruses likely pre-dates the divergence of the Archaea and Eukarya (Prangishvili et al., 2017; Krupovic et al., 2018), a robust archaeal virus model system could provide new insights into the evolution of virus lineages and viral replication strategies as well as mechanisms of viral virulence, host resistance, and virus attenuation.

The Sulfolobus Spindle-shaped Virus (SSV) system has become a popular model for studying thermophilic archaeal virus biology and virus-host biogeography. Several factors make this system ideal for studying virus-host infections in crenarchaea (i.e., Sulfolobales). First, endemic populations of SSV hosts from the family Sulfolobaceae exhibit biogeographic structure such that there is a positive correlation between genetic distance among strains (i.e., divergence) and geographic distance among various sites from which strains have been isolated (Grogan, 1989; Whitaker et al., 2003; Reno et al., 2009). SSVs also exhibit biogeographic structure on a global-scale (Held and Whitaker, 2009). Second, the highly acidic $(\mathrm{pH}<4.5)$ and high temperature $\left(65-88^{\circ} \mathrm{C}\right) \mathrm{SSV}-S u l f o l o b u s$ habitats have low biodiversity, limiting the potential for host switching, which can confound efforts to elucidate the genetic underpinnings of virus-host infection profiles (Munson-McGee et al., 2018). Third, SSVs and Sulfolobales can be readily cultured both in liquid media (e.g., yeast-sucrose, tryptone) and on gellan gum (e.g., GelRite $\left.{ }^{\circledR}\right)$ plates (Zillig et al., 1996; Stedman, 2008; Ceballos et al., 2012). Fourth, given the wide geographical separation between sulfuric hot springs, which are habitats for SSVs and hosts, this system is amenable to studying multiple allopatric and sympatric virus-host pairs (Ceballos et al., 2012), which is essential for studying virus-host interactions, biogeography, and coevolution (Greischar and Koskella, 2007).

SSVs comprise the Fuselloviridae (International Committee on Taxonomy of Viruses, 2017) with SSV1 being the founding archetype of this family of "little spindle" (Latin: fusello) shaped viruses (Martin et al., 1984; Reiter et al., 1987; Palm et al., 1991). SSV1 was isolated from Sulfolobus shibatae strain B12, which was derived from a sulfuric hot spring in Beppu, Japan (Martin et al., 1984; Grogan et al., 1990). The SSV1 virion was shown to harbor a positively-supercoiled double-stranded (ds) DNA genome (Nadal et al., 1986) and virion production was shown to be UV inducible (Martin et al., 1984; Schleper et al., 1992). Studies also demonstrated that, apart from the fully assembled virion, SSV1 could reside either episomally, as a positively-supercoiled, negatively-supercoiled, or relaxed dsDNA viral genome within the host (Nadal et al., 1986); or, fully integrated (often in tRNA genes) in the host genome as a provirus (Reiter et al., 1989).

Soon after the initial characterization of SSV1, other SSVs were isolated and characterized from geothermal regions worldwide. Until two recent publications that expand the number of characterized genotypes (Pauly et al., 2019; Zhang et al., 2020), only ten SSVs had been well-characterized with six of these strains: SSV1, SSV2, SSV3, SSV8 (a.k.a., SSVRH), SSV9 (a.k.a., SSVK1), and SSV10 (a.k.a., SSVL1) being the most extensively studied (for review see Goodman and Stedman, 2018). These SSVs have genomes that range from about 14.7$17.5 \mathrm{kbp}$ (depending on the strain) consisting of 32-36 openreading frames (ORFs) featuring polycistronic transcription units. Assembled SSV particles have a major axis of $\sim 80-100 \mathrm{~nm}$ and a minor axis of $\sim 60 \mathrm{~nm}$.

For over three decades, SSVs have been reported to be nonlytic budding viruses that infect Sulfolobus (Martin et al., 1984; Reiter et al., 1987; Palm et al., 1991; Schleper et al., 1992; Zillig et al., 1998; Wiedenheft et al., 2004; Contursi et al., 2006; Prangishvili et al., 2006; Ceballos et al., 2012; Fusco et al., 2015; Quemin et al., 2016). The term non-lytic refers to the fact that SSV infection results in inhibition of cell growth (both in liquid culture and on lawns) rather than the gross lysis of host cells and cell death in liquid culture or clear plaques on host lawns-both of which result from lytic replication. Recently, it was suggested that SSV9 can induce a state of dormancy, empty cells, and eventual host death in a sympatric host; however, the mechanism for this proposed dormancy is unclear and there does not seem to be any cell lysis (Bautista et al., 2015).

Plaque-like "halo" assays using both sympatric and allopatric hosts have repeatedly shown that different SSVs (e.g., SSV1, SSV2, SSV3, SSV8, and SSV10) form turbid areas of growth inhibition or halos on host lawns (Figures 2A, B), often featuring diffuse boundaries (Martin et al., 1984; Schleper et al., 1992; Wiedenheft et al., 2004; Ceballos et al., 2012; Iverson and Stedman, 2012). Yet, halo assays using SSV9 (formerly known as SSVK1), isolated from the Valley of Geysers (Russian: Долина гейзеров) Kamchatka, Russia (Wiedenheft et al., 2004), form large clear plaques on host lawns (Figure 2C) in contrast to the turbid diffusely-bound halos characteristic of all other SSVs (Ceballos et al., 2012), indicating that SSV9 may replicate differently than the other SSVs (Bautista et al., 2015).

To test the hypothesis that SSV9 lyses susceptible hosts within the family Sulfolobaceae, host growth profiles in singlevirus/single-host infection assays using three distinct SSVs (i.e., SSV1, SSV8, and SSV9) were evaluated. Liquid culture and spoton-lawn "halo" assays were used to verify productive infection by a given SSV. To assess the relative susceptibility of different hosts to a given SSV and to elucidate conspicuous differences in host growth profiles during the infection, both allopatric and sympatric hosts were infected. Each specific virus-host pairing 
was conducted under different culture conditions to evaluate the impacts of distinct conditions on growth dynamics. For example, different multiplicities of infection (MOI) were tested and end-point assays (in liquid culture) were conducted to measure virus and host dynamics in parallel. In addition to evaluating halo "phenotypes" in plate-based assays, small-scale liquid culture assays were conducted to gauge the relative amount of cellular debris emerging from different virus-host pairings. Assays to assess the relative stability of different SSVs under conditions that simulate the natural Sulfolobales habitats were also performed to determine if virion stability plays a role in successful transmission and between strain differences in virion production.

\section{MATERIALS AND METHODS}

\section{Virus Preparation}

Glycerol stocks of SSV-infected Sulfolobus strains stored at $-80^{\circ} \mathrm{C}$ were partially thawed on ice. For most trials, $100 \mu \mathrm{L}$ of infected cell suspension were added to $30 \mathrm{~mL}$ of YS media $(\mathrm{pH}$ 3.2) in a $125 \mathrm{~mL}$ Erlenmeyer flask (per Ceballos et al., 2012). Tryptone (T) or tryptone-sucrose (TS) media was used in select experiments by substituting $3.0 \mathrm{~g} \mathrm{~L}^{-1}$ tryptone for yeast and sucrose or $2.0 \mathrm{~g} \mathrm{~L}^{-1}$ tryptone plus $1.0 \mathrm{~g} \mathrm{~L}^{-1}$ sucrose for yeast, respectively. Flasks were loosely capped and incubated at $78^{\circ} \mathrm{C} / 90$ RPM shaking.

Once liquid culture reached an optical density $\left(\mathrm{OD}_{600}\right)$ between 0.4 and $0.6,3.0 \mathrm{~mL}$ of cell suspension was used to inoculate $600 \mathrm{~mL}$ of pre-heated fresh media in a $1.0 \mathrm{~L}$ baffled flask.

These cultures were incubated at $78^{\circ} \mathrm{C} / 70 \mathrm{RPM}$ until reaching an $\mathrm{OD}_{600}=0.6-0.8$ to maximize virus yields. The culture was subsequently centrifuged for $20 \mathrm{~min}$ at 6,000 RPM (Sorvall RT Legend Centrifuge, Fiberlite $4 \times 800 \mathrm{~mL}$ fixed-angle rotor with $250 \mathrm{~mL}$ inserts; ThermoFisher, Pittsburgh, PA) to pellet the cell mass while leaving virus in suspension. The supernatant was decanted and filtered through a $0.45 \mu \mathrm{m}$ vacuum PES filter system. The filtrate was concentrated using $10 \mathrm{kDa}$ Centricon Plus-70 spin concentration tubes (Millipore Corp., Billerica, MA, USA) to produce $\sim 3.0 \mathrm{~mL}$ concentrated SSV suspension.

TEM was used to confirm the presence of virions and virus particle count was measured using electrospray ionization/mass spectrometry or serial dilution halo assays (see below). Virus was stored at $4^{\circ} \mathrm{C}$ and used in spot-on-lawn "halo" assays and liquid culture infection assays within $2-3$ weeks of harvesting. Dilutions of viral stocks were used as inocula.

\section{Host Cell Preparation}

Glycerol stocks of uninfected Sulfolobus strains stored at $-80^{\circ} \mathrm{C}$ were used to establish $30 \mathrm{~mL}$ cultures in YS (or T or TS) media, as described above. Uninfected Sulfolobus cultures were either used to prepare host lawns for halo assays (Ceballos et al., 2012) or used in liquid culture infection assays (as described below).

\section{Transmission Electron Microscopy (TEM) of Viruses and Cells}

To verify the presence of SSVs in samples, $\sim 5 \mu \mathrm{L}$ of viral suspension was spotted onto a formvar-coated copper grid and incubated for $10 \mathrm{~min}$ in a humidity chamber. The sample was rinsed with distilled water and negatively stained with a $1 \%$ solution of uranyl acetate for $30 \mathrm{~s}$. The stain was wicked off the grid and then the sample was air dried. Grids were imaged with a Hitachi H-7100 TEM at $75 \mathrm{kV}$. Images were captured at 60,000$200,000 \times$ magnification. TEM images for cells were acquired as described in Brumfield et al. (2009). Cells were fixed in glutaraldehyde $(3 \% \mathrm{v} / \mathrm{v})$, centrifuged, and re-suspended in a small volume of agar $(2 \% \mathrm{v} / \mathrm{v})$. After solidification, the resulting agar was cut into small pieces and fixed overnight with glutaraldehyde $(3 \% \mathrm{v} / \mathrm{v})$ in $0.05 \mathrm{M}$ potassium sodium phosphate buffer (PSPB) and $\mathrm{pH}=7.2$. Agar pieces were rinsed twice for $10 \mathrm{~min}$ each with PSPB. Agar pieces were post-fixed with osmium tetroxide $(2 \% \mathrm{v} / \mathrm{v})$ at RT for $4 \mathrm{~h}$. Samples were dehydrated via an ethanol rinse series (50\%-100\% v/v) and then washed with a transitional solvent, propylene oxide. Spurr's resin (Spurr, 1969) was used to infiltrate dehydrated cell mass and pieces were baked overnight at $70^{\circ} \mathrm{C}$. Thin sections $(60-90 \mathrm{~nm})$ were cut and stained with uranyl acetate and lead citrate. Imaging was done with a LEO 912AB TEM.

\section{Electrospray lonization/Mass Spectrometry (ESI/MS)}

Virus suspension preparations were processed in an Integrated Viral Detection System (BVS, Inc; Missoula, MT) per protocols in Wick and McCubbin (1999). A mixture consisting of $100 \mu \mathrm{L}$ viral suspension and $900 \mu \mathrm{L}$ ammonium acetate buffer solution is aerosolized in a Electrospray Aerosol Generator (Model 3480, TSI, Inc., Minnesota). A Differential Mobility Analyzer (Model 3081, TSI, Inc., Minnesota) separates particles by their electrical mobility, which is influenced by particulate mass-to-charge ratio or "size." These particles flow in tandem with a saturated butanol fluid. The particles initiate butanol condensation and the stream is cooled enabling butanol-condensed particles to be optically counted in a Condensate Particle Counter (Model 3772, TSI, Inc., Minnesota). System software displays results in terms of particle count per size category with a standard range of 2-280 $\mathrm{nm}$.

This specialized ESI/MS is designed to detect intact virus particles (Wick et al., 2006) and can measure relative virus particle count between two or more samples. In these infection assays, ESI/MS spectra were evaluated by using either peak values or the area under the curve to compare SSV particle production between two cultures.

Note: Time-of-flight estimation based on mass: charge is used to calculate ESI/MS “size." ESI/MS "size” will differ from physical size as measured by other methods, such as transmission electron microscopy. In addition, mass:charge size vs. physical size will differ due to the assumption of virus particle sphericality in time-of-flight derivations. SSV s have been shown under electron microscopy to be fusiform or "spindle-shaped" particles of $\sim 60$ $\times 90 \mathrm{~nm}$ (and not spherical), thus, a discrepancy is expected. In some cases, SSVs exhibited dual peaks or shouldered peaks in the ESI/MS analyses. Such spectral features may be due to different conformations of the same SSV or, potentially, due to the presence of pleomorphic SSVs. Under ESI/MS, SSVs exhibit characteristic peaks between 46.1 and $61.5 \mathrm{~nm}$ for purified virus 
suspensions with major peaks typically appearing at 46.1, 47.8, and 49.6 within the $\pm 4 \mathrm{~nm}$ system tolerance.

\section{Spot-on-Lawn Halo Assays}

Halo assays were performed (per Stedman et al., 2003) and cultures were grown as described above. At an $\mathrm{OD}_{600}=0.4-$ $0.6,500 \mu \mathrm{L}$ of cell suspension were added to $4.5 \mathrm{~mL}$ of a $78^{\circ} \mathrm{C}$ mixture of equal parts $1.0 \% \mathrm{w} / \mathrm{v}$ Gelrite ${ }^{\circledR}$ (Sigma-Aldrich, St. Louis, MO, USA) and 2-fold concentrated YS medium. The $5 \mathrm{~mL}$ mixture was spread on pre-warmed Gelrite ${ }^{\circledR}$ plates $(1 \% \mathrm{w} / \mathrm{v}$ in medium) and allowed to solidify for $15 \mathrm{~min}$ at room temperature followed by a $20 \mathrm{~min}$ incubation at $78^{\circ} \mathrm{C} .1 .0 \mu \mathrm{L}$ of each viral suspension was spotted onto the prepared plate in labeled areas. Depending upon host strain, plates were incubated for 3-9 days at $78^{\circ} \mathrm{C}$. Successful infection was scored by the formation of a visible halo of growth inhibition on the host lawn. Triton X-100 $(0.05 \% \mathrm{v} / \mathrm{v})$ was used as a positive control and sterile water was used as a negative control. Initial tests also used a $2 \mu \mathrm{L}$ spotting of ultrafiltrate $(10 \mathrm{kDa} \mathrm{MWCO})$ to ensure that effects were not from other proteinaceous toxins (e.g., sulfolobicins) but from SSV infection.

\section{Liquid Culture Infection Assays}

$3.0 \mathrm{~mL}$ of uninfected Sulfolobus culture were diluted 1:100 in preheated media in a $1.0 \mathrm{~L}$ baffled flask. Cultures were grown to an $\mathrm{OD}_{600} \approx 0.15$. Then, $100 \mu \mathrm{L}$ of standardized viral suspension were added to the flask. Sulfolobus cultures were incubated with shaking $\left(78^{\circ} \mathrm{C} / 70 \mathrm{RPM}\right)$ for various time intervals. Virus was then harvested as detailed above and ESI was used for virus particle counts. Growth curves from SSV-Sulfolobus cultures were fit to Logistic, modified Logistic (Equations 1, 2), Gompertz, modified Gompertz (Equations 3, 4), and other growth models (Gompertz, 1825; Laird, 1965; Zwietering et al., 1990; Lopez et al., 2004; Sprouffske and Wagner, 2016):

$$
\begin{array}{r}
y(t)=\frac{A}{1+\left(\frac{A-y_{0}}{y_{0}}\right) e^{-r t}} \\
y(t)=\frac{A}{1+\exp \left(\frac{4 \mu}{A}(\lambda-t)+2\right)} \\
y(t)=A \cdot \exp \left[-\ln \left(\frac{y_{0}}{A}\right) \cdot \exp (-\mu \cdot t)\right] \\
y(t)=A \cdot \exp \left[-\exp \left(\frac{\mu \cdot \exp (1)}{A}(\lambda-t)+1\right)\right]
\end{array}
$$

The exp represents an exponential function such that $\exp (\mathrm{x})=$ $\mathrm{e}^{\mathrm{x}}$, where $\mathrm{e}$ is Euler's number. $A$ represents the amplitude or peak growth value in the given environment, which corresponds to stationary phase and the maximum carrying capacity; $r$ is the intrinsic growth rate coefficient; $y_{0}$ is the initial population size; $\mu$ is the maximum slope of the growth curve, and $t$ is time. For equations in which the maximum specific growth rate $\left(\mu_{\max }\right)$ was not a fit parameter; it was calculated by taking the derivative of $y(t)$ evaluated at a software-derived point of maximum growth using parameter estimates for $\mathrm{A}, r$, and $y_{0}$.

Parameter estimates were derived in R 3.6.1 (R Core Team; R Foundation for Statistical Computing, Vienna, Austria) using curve fitting functions of easynls, growthcurver, and grofit packages (Kahm et al., 2010; Sprouffske and Wagner, 2016; Arnhold, 2017). The best parameter values for each function were found via Levenberg-Marquardt non-linear least-squares algorithm (Moré, 1978). The levels of fit between software packages varied and provided slightly different parameter values for each dataset. Relative area under the curve values, however, remained consistent across assessments within the same fitted models (i.e., Gompertz and Gompertz with software "A") and with direct calculation of Riemann sums of integral of curves. The area under the optical density-based growth curves was calculated via two methods for each data set. The fitted models were integrated from the time of infection $(t=0)$ to the timepoint delineating the end of the exponential growth phase of the uninfected host growth curve (i.e., the control). This process was used for each data set resulting in successful fits to Equations (1)(4) (above) as well as other growth models (not shown here). The second method for calculating AUC values was through Riemann sums, also known as the trapezoidal approximation method (Hasenbrink et al., 2005). Equation (5) shows the formula utilized for calculating the AUC with this method.

$$
A U C_{\text {Riemann }}=\sum_{i=1}^{n-1}\left(\frac{y_{i}+y_{i+1}}{2} \times\left(t_{i+1}-t_{i}\right)\right)
$$

Area under the curve values for virally challenged hosts were then standardized to the AUC value for the averaged uninfected host control growth curve in order to calculate the percent inhibition (PI) for the individual growth curves (see Tables S1-S3). The equation used to calculate the percent inhibition of growth is shown in Equation (6) below, from Rajnovic et al. (2019) which is reformatted in Equation (7). For a full discussion of this method (see Ceballos and Stacy, under review).

$$
\begin{array}{r}
P I=\frac{A U C_{\text {control }}-A U C_{\mathrm{inf}}}{A U C_{\text {control }}} \\
P I=1-\frac{A U C_{\mathrm{inf}}}{A U C_{\text {control }}}
\end{array}
$$

The left and right bounds of the integration are key parameters affecting the area under the curve metric. For these viral growth curves, the point of first difference is at time $t=0$ when virus is added. The upper bound of the growth curve was originally chosen to be the intercept of the line produced by the maximum growth rate and the fitted asymptotic value. This method was not used because it does not include the transition period between the exponential growth phase and the stationary phase of growth, an area of the growth curve which we considered to be worthy of inclusion. Datapoints at and beyond stationary phase were excluded from AUC analysis because they disproportionately value the asymptotic (A) parameter, which undermines the robustness of the AUC value as a unifying metric of growth curve changes.

Virus replication data for SSV8 were fit to an exponential growth model both based on peak values and AUC calculations (with similar patterns emerging with either approach):

$$
y(t)=A e^{(t / \tau)}
$$


Parameter $\mathrm{A}$ is the initial population prior to infection and $1 / \tau$ is the growth rate constant.

For SSV9-infected cultures, average OD-based growth was fit to a sinusoidal function:

$$
y(t)=A e^{(-t / \tau)} \cdot \sin (\omega t)
$$

Term $A e^{-t / \tau}$ represents the exponential decay of the oscillation amplitude whereby $\mathrm{A}$ is the driving amplitude and $1 / \tau$ is a decay rate constant. In the second term, $\omega$ is the frequency variable. The average growth curve of SSV9-infected cultures was fit to a damped sinusoidal function simply because this function best described the cyclical bursts in growth that had increasing smaller amplitude and which were each followed by rapid declines in host population (as determined by optical density readings). However, for SSV9 replication, a Gaussian function was used to fit the dataset.

$$
y(t)=A e^{-\frac{\left(t-t_{c}\right)^{2}}{2 w^{2}}}
$$

Parameter A represents the amplitude of the peak function; $t_{c}$ is the time at which the center of the peak occurs; and, w represents the width of the peak function at one-half the peak amplitude. The "goodness-of-fit" of these data sets (assessed by adjusted $r^{2}$ values) to the sinusoidal and Gaussian, respectively, prompted their use. For the sinusoidal fit, the adjusted $r^{2}$ value was 0.90 (see Figure S2B). For the Gaussian fit to the SSV9 virus replication data, adjusted $r^{2}=0.94$. Note that $r^{2}$ values typically were between 0.90 and 0.99 , except for fits to the SSV9-infected host growth curves, which failed or had $r^{2}<0.25$.

\section{qPCR for Determination of SSV9 Genome Copy Number}

SYBR green-based qPCR was used to quantify the number of SSV9 genomes for inocula and end-point infection. Two PCR primers were employed: SSV9F (5'-GTGAAGCGACCA ACATAGGTGCAA-3') and SSV9R (5'-GTTGCGTTTGTACCG GTTACGCTA-3') -targeting the single-copy gene $v p 1$, which encodes a major SSV structural protein (Bautista et al., 2015). Standard curves were generated with 10 -fold serial dilutions $\left(10^{8}-10^{0}\right.$ copies) of the $v p 1$ gene fragment $(138-\mathrm{bp})$ cloned into a TOPO TA pCR2.1 plasmid (Invitrogen). Copy number in each standard was calculated using formula:

$$
\begin{aligned}
& \text { gene copy number }= \\
& \frac{\left[\text { sample concentration }\left(\frac{n g}{\mu l}\right)\right] \cdot N_{A}}{[\text { fragment length }(b p)] \cdot 1 \times 10^{9}\left(\frac{n g}{g}\right) \cdot 660(\mathrm{~g} / \mathrm{mol})} .
\end{aligned}
$$

where, $660 \mathrm{~g} / \mathrm{mol}$ is the molecular weight of one base pair, $N_{A}$ is Avogadro's number, and $1 \times 10^{9}$ is used to convert units to nanograms. Each qPCR reaction was performed on a Rotor-Gene Q (Qiagen, Inc.; Maryland, USA) and consisted of $1 \times$ Green/ROX qPCR Master Mix (Fermentas, Inc.; Ontario, Canada), 3 pmol of each primer, $1 \mathrm{uL}$ of DNA template, and nuclease-free water to a final volume of $20 \mathrm{~mL}$. Three technical replicates were performed of each standard and samples. The qPCR cycle parameters were as follows: $98^{\circ} \mathrm{C}$ for $2 \mathrm{~min}$, followed by 40 cycles of $98^{\circ} \mathrm{C}$ for $5 \mathrm{~s}$ and $20 \mathrm{~s}$ at $60^{\circ} \mathrm{C}$. A melt curve analysis was performed after each run from 65 to $95^{\circ} \mathrm{C}$ in $0.5^{\circ} \mathrm{C}$ increments at $2 \mathrm{~s}$ intervals, to ensure specific amplification of a single target, and no primer dimer formation. The $\mathrm{qPCR}$ amplicons were resolved in $2 \%$ agarose gels to assess amplicon sizes for target-specific amplification. Reaction mixtures with sterile water (no-template DNA) or pCR2.1 DNA without insert served as a negative controls to control for false positives. Assays showed amplification efficiencies of $100 \% \pm 10 \%$ (i.e., with a slope between -3.6 and -3.1$)$, consistency across replicate reactions, and linear standard curves $\left(R^{2}>0.970\right)$. The absolute quantification method was used to calculate the number of viral genomes per $\mathrm{mL}$ of culture.

\section{RESULTS}

\section{Virus Yields and Confirmation of SSV Infection}

To ensure that virus infection was a cause of host growth decline, presence of virus was determined by one (or more) of four distinct methods: electrospray ionization/mass spectrometry (ESI/MS) in units of virus particles per milliliter $\left(\mathrm{VP} \mathrm{mL}^{-1}\right)$; serial dilution plaque-like assays in units of halo-forming units per milliliter ( $\mathrm{hfu} \mathrm{mL}^{-1}$ ); virus-like particle (VLP) counts using transmission electron micrographs (VLP $\mathrm{mL}^{-1}$ ); and/or, quantitative polymerase chain reaction (qPCR) in terms of the total number of viral genomes per milliliter $\left(\mathrm{v}_{\mathrm{g}} \mathrm{mL}^{-1}\right)$. For infection assays, end-point titers were also determined by one or more of these methods. ESI/MS and plate assays provided the most consistent results.

Growing $600 \mathrm{~mL}$ liquid cultures of permissive host, each infected with a single SSV strain, to late $\log$ phase growth (e.g., $\mathrm{OD}_{600 \mathrm{~nm}}=0.8=5.4 \times 10^{8}$ cells $\mathrm{mL}^{-1}$ ) resulted in comparable virion yields as shown by electrospray ionization/mass spectrometry (ESI/MS) spectra (Figure S1). For this culture volume, SSV8 and SSV9 suspensions result in $\sim 2 \times 10^{8}$ to $4 \times 10^{8} \mathrm{VP} \mathrm{mL}{ }^{-1}$. SSV1 yields are typically $50-80 \%$ lower. Thus, 1.2-2.0 L of SSV1-infected culture were required to ensure stocks were the same order of magnitude and comparable to SSV8 and SSV9. SSV suspensions were then diluted to produce working stocks with equivalent titers (e.g., $\sim 1 \times 10^{8} \mathrm{VP} \mathrm{mL}^{-1}$ ). Verification of the presence of SSV virions in virus suspensions was accomplished by transmission electron microscopy (Figure 1). Electron micrographs typically feature SSV particles either in rosettes, where multiple virons are attached at one end presumably via electrostatic and hydrophobic interactions (Quemin et al., 2015) between tail fibers (see Figure 1, right panel), or to cell debris (e.g., membrane remnants) by their tail fibers (see Figure 1, left and middle panels). Depending upon the host in which the SSVs are cultivated, slight changes in morphology (i.e., elongation) may present. However, SSV9 consistently shows a more elongated virion (Figure 1, right panel).

It has been previously reported that not all species within the family Sulfolobaceae are susceptible to SSV infection (Ceballos et al., 2012). Thus, to ensure that there was active infection in the virus-host pairings selected for this study, two validation 


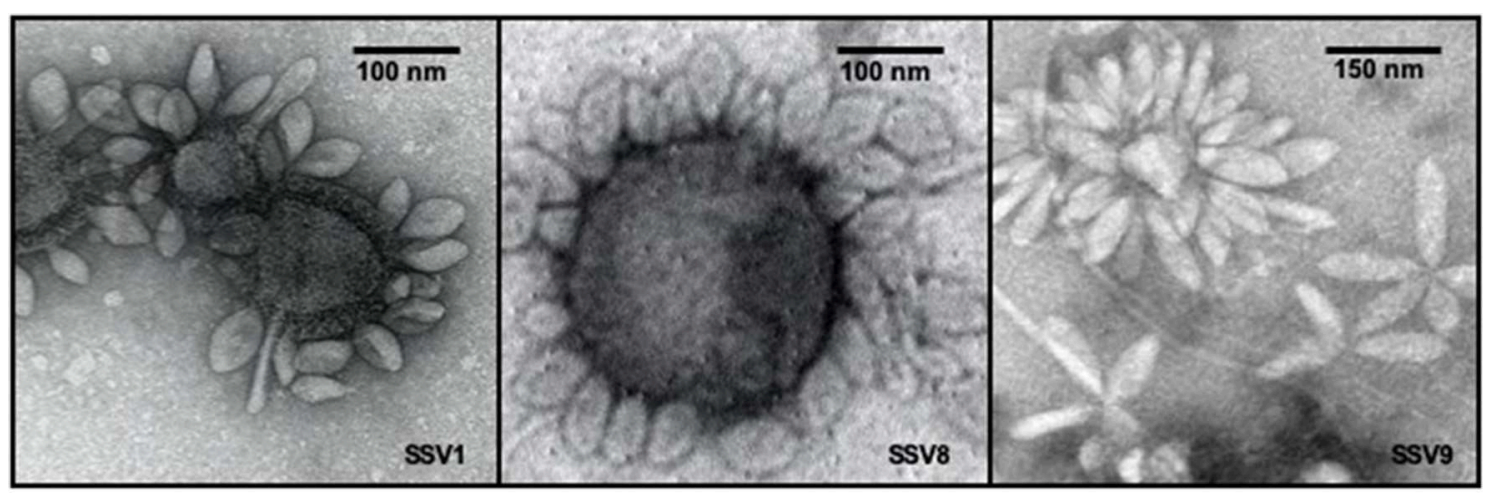

FIGURE 1 | Transmission electron micrographs of SSV particles. Transmission Electron Microscopy (TEM) demonstrates the presence of spindle-shaped virus particles after harvest and concentration steps at end-point of infection assays as well as during the preparation of virus stocks for liquid culture infection assays and halo assays. TEM images of SSV1 (left), SSV8 (middle), and SSV9 (right).

steps were taken. First, spot-on-lawn plate assays were used as a qualitative (i.e., yes or no) verification that a given SSV strain is able to infect a specific strain of the family Sulfolobaceae (Figure 2). Second, small-scale test infections in liquid culture were performed and TEM images were obtained (Figure 3).

To characterize halo phenotype and validate SSV infectivity, $2 \mu \mathrm{L}$ of virus suspension was used in spot-on-lawn plate assays. On multiple susceptible hosts (see Ceballos et al., 2012), SSV1 consistently induces a smaller more diffuse halo phenotype (Figure 2A). The halo phenotypes induced by SSV2 and SSV3 vary depending upon the host (data not shown) and are less consistent than those produced by SSV1 and SSV8. SSV8 consistently produces large halos on hosts with diameters comparable to those produced by SSV9. However, SSV8 halos have diffuse boundaries (Figure 2B); whereas SSV9 boundaries are sharp (Figure 2C). To control for sulfolobicin-induced halos (false positive SSV infection), negative controls using ultrafiltrate (10 kDa MWCO) were also spotted on lawns as a negative control. Sulfolobicins are reported to be $\sim 20 \mathrm{kDa}$ (Prangishvili et al., 2000).

Together spot-on-lawn plate assays and electron micrographs from liquid culture infections validate whether a specific SSV strain is able to productively infect a putative host strain. Productive SSV1, SSV2, SSV3, SSV8, SSV9, and SSV10 infection on host strain G $\theta$ was confirmed through these procedures prior to conducting large-scale liquid culture assays. For large-scale trials, we used only SSV1, SSV8, and SSV9 because of consistency in halo phenotypes and due to the fact that SSV8 exhibits one of the highest levels of relative virulence (and, thus is a comparator for SSV9) across multiple susceptible hosts.

\section{Host-Growth After Infection by Various SSVs}

Liquid culture assays using SSV1, SSV2, SSV3, SSV8, and SSV10 infecting the universally susceptible host strain Sulfolobus strain $\mathrm{G} \theta$ (Cannio et al., 1998), reveal that host growth $\left(\mu_{\max }\right)$ is slowed and the peak cell density upon entering the stationary phase (i.e., $N_{\text {asymptote }}$ ) is reduced compared to uninfected control (Figure 4). Individual (i.e., single virus-single host) infections with SSV1,
SSV8, or SSV10 on strain G $\theta$ show archetypal host growth with these reportedly non-lytic SSVs. Infection with either of two SSVs isolated from two geographically-distinct Icelandic geothermal regions-SSV2 (from Reykjanes) and SSV3 (from Krisovic) also show reductions in $\mu_{\max }$ and area-under-the curve (AUC) when compared to controls. Despite SSV2 and SSV3 infections exhibiting slightly different host growth profiles (Figure 4A, cyan and mustard lines) due to slower growth to stationary phase, data still fit a Gompertz model (Gompertz, 1825; Laird, 1965) with high coefficient of determination values (Table S1). Measures of percent inhibition were derived by AUC calculations (Zwietering et al., 1990) for the infected host growth and dividing by the AUC for the uninfected control (Figure 4B). Using this quotient, an index of relative virulence $\left(V_{R}\right)$ between strains on a given host was generated.

Using a Gompertz model and AUC as a measure of percent inhibition (PI), SSV1 exhibits the lower PI (8.57\%) while SSV8 has a significantly higher PI $(28.44 \%)$ with $\mathrm{R}^{2}$ values for the Gompertz of 0.9875 and 0.9775 , respectively. Employing Riemann Sums or alternative models (i.e., Logistic Growth) does not significantly alter relative virulence $\left(V_{R}\right)$ for SSV1 and SSV8 (see Table S1). Regardless of analytical approach, the general trend in virulence of SSVs on Sulfolobus strain GO is: SSV1 < SSV10 $<<$ SSV2 $\approx$ SSV3 $<$ SSV8. Note that AUC measures suggest that SSV2 and SSV3 have a slightly greater PI than SSV8. However, note that AUC for SSV2 and SSV3 is underestimated due to a lag in both strains reaching stationary phase compared to the other SSVs (Ceballos and Stacy, under review). Therefore, in subsequent trials, SSV1 and SSV8 were chosen as comparators for SSV9.

\section{Host-Growth After Infection With SSV9}

Unlike infection with SSV1, SSV2, SSV3, SSV8, or SSV10, Sulfolobus strain G $\theta$ infected with SSV9 did not grow with Gompertzian-like (Gompertz, 1825; Laird, 1965) dynamics. In contrast, rapid and significant inhibition of culture growth was observed in cultures infected with SSV9. This pattern was observed for several Sulfolobales strains over multiple replicates $(3 \times$ per trial) with each strain as shown in Figure 5 and 


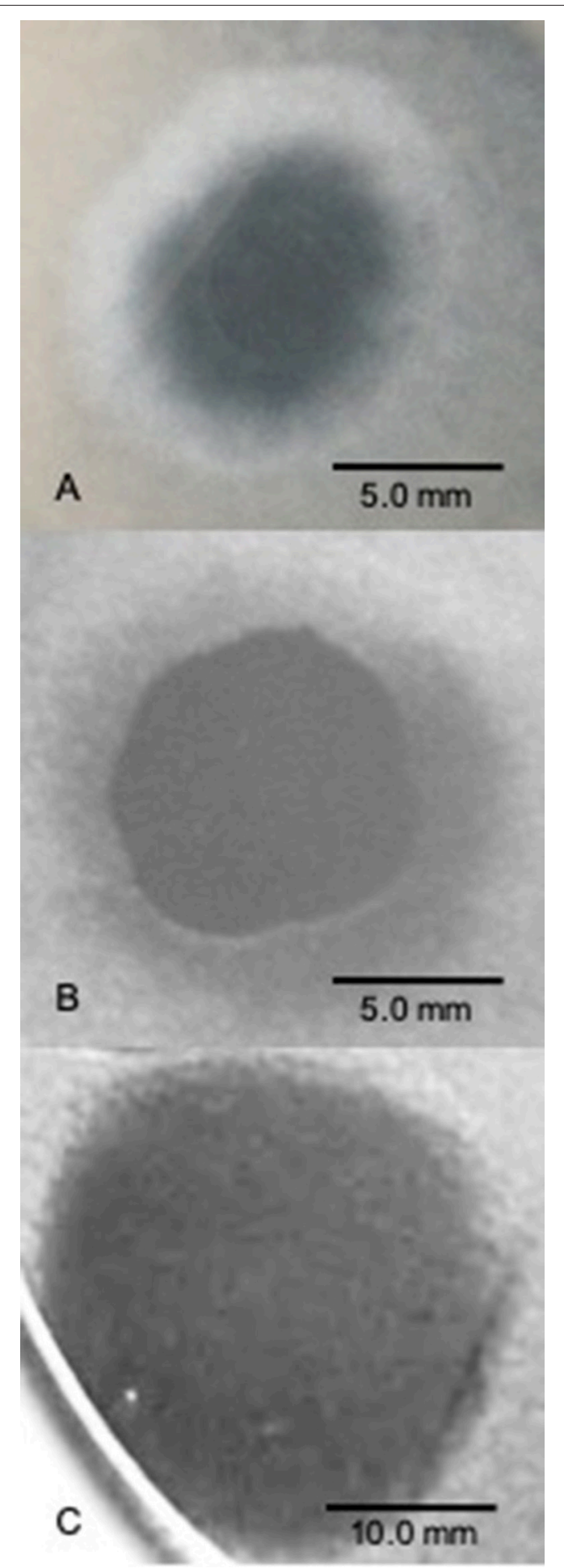

FIGURE 2 | SSV spot-on-lawn halo assays. A $2 \mu \mathrm{L}$ drop of virus suspension was spotted on lawns of Sulfolobus solfataricus strain $\mathrm{G} \theta$ to determine whether a viral infection can be established and the nature of the virus-host interaction in terms of halo "phenotype" (size, shape, boundary type). (A) SSV1 forms broad diffuse, turbid halo. (B) SSV8 (a.k.a., SSVRH) makes a broad diffuse, turbid halo with a sharper boundary between the direct application point and halo leading edge; (C) SSV9 (a.K.a., SSVK1) yields a large and complete clearing of host lawn.

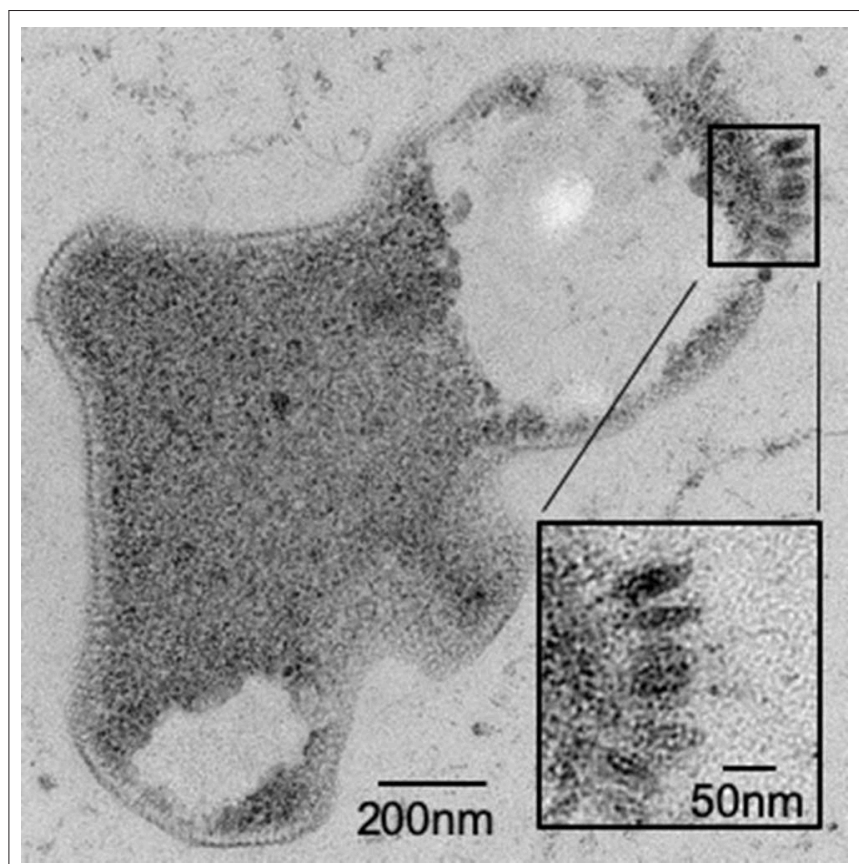

FIGURE 3 | Sulfolobus strain G $\theta$ infection by SSV verified by TEM.

Transmission Electron Micrograph (TEM) of a 30-60 $\mu \mathrm{m}$ epoxy-impregnated section $(60,000 X$ magnification) from a host cell infected with SSV8. A closer view (inset) of this micrograph reveals fusiform or "spindle-shaped" virus-like particles on the surface of the cell membrane.

multiple trials $(2-4 \times)$ for each virus-host pairing. Furthermore, all susceptible Sulfolobales strains tested serve as allopatric hosts to SSV9.

Infection assays using SSV1 (Beppu, Japan), SSV8 (Yellowstone National Park, USA), and SSV9 (Valley of Geysers, Kamchatka, Russia) were used to infect susceptible hosts: S200 (a.k.a., S. icelandicus HVE 10/4 from the Hveragerdi thermal region of Iceland); S444 (an isolate derived from Lassen Volcanic National Park, California in the USA); and, S437 (a.k.a, DSM1617; isolated from a hot spring near Pisciarelli, Italy). With the exception of SSV1 infection of strain HVE 10/4, all host growth curves show significantly reduced $N_{\text {asymp }}$, AUC, and/or $\mu_{\max }$ for SSV1 and SSV8 when compared to uninfected controls. However, when each host was infected with SSV9, a distinct host growth profile emerged. Specifically, Gompertzian Models failed to adequately represent the SSV9 infection data. Instead, non-Gompertzian cyclical spike patterns are consistently observed in host growth. Low $R^{2}$ values $(<0.5)$ emerged for both individual traces and averaged growth curves for SSV9 (Figure 5, red traces), whereas, high $R^{2}$ values $(>0.9)$ were typical for all other SSVs infecting these same hosts (Table S2).

\section{Host-Growth Profile of SSV9 Is Not MOI-Dependent}

To determine if the unusual growth profiles of SSV9-infected hosts are MOI dependent, dilutions of SSV8 and SSV9 stocks were used in liquid culture assays on host strain G $\theta$ (Figure 6).

The typical host growth pattern shown for SSV infection (i.e., Gompertzian-like growth) is observed for all SSV8 infections on 

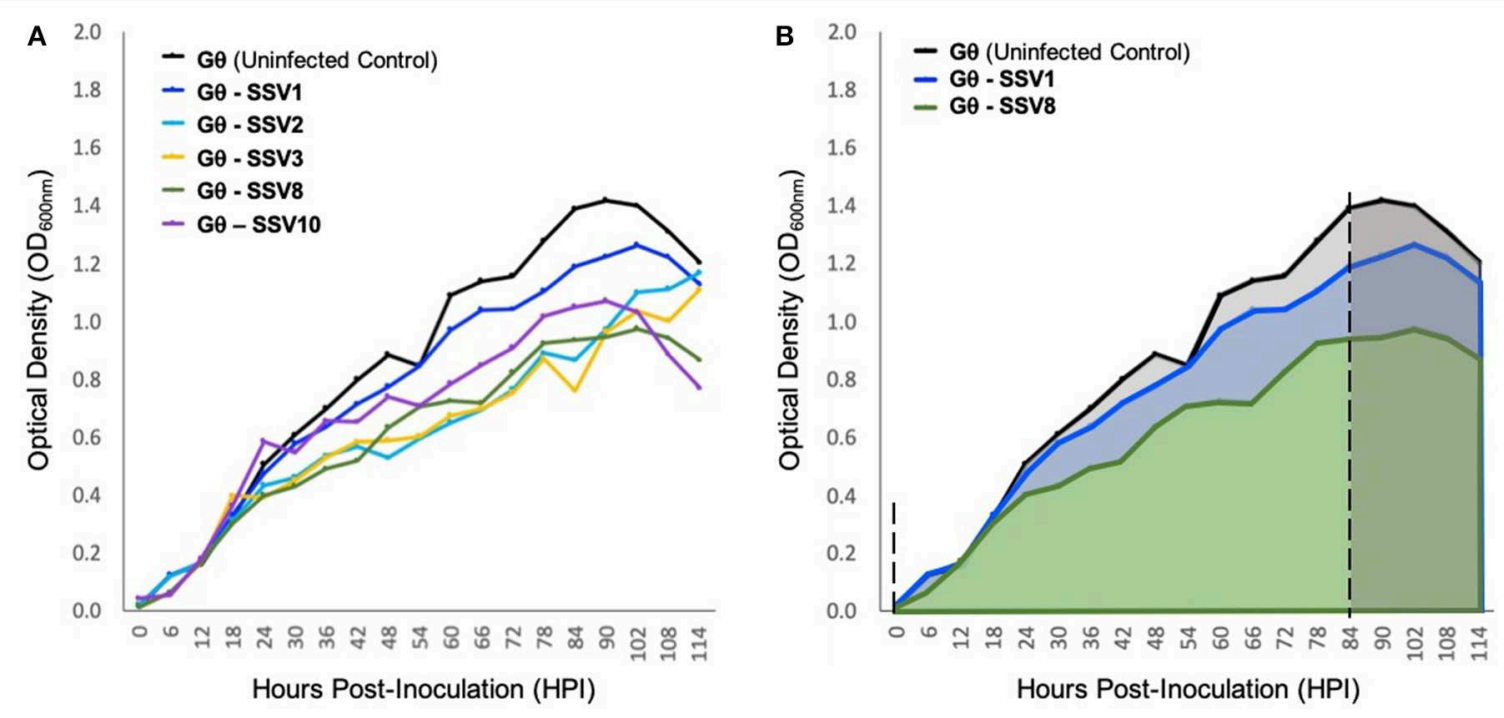

FIGURE 4 | Growth of host strain Sulfolobus G $\theta$ during SSV infection. (A) Sulfolobus strain G $\theta$ infected with SSV1, SSV2, SSV3, SSV8 (a.k.a., SSVRH), and SSV10 (a.k.a., SSVL1) at a MOI $=0.01$. (B) Graphical representation of percent inhibition of Sulfolobus strain Ge via area-under-the-curve (AUC). All growth curves fit with high coefficients of determination ( $R^{2}$ values) to Gompertz Models or Logistic Growth Models (see Table S1). The asymptotes (dashed) indicate point of inoculation until end of the exponential growth phase/onset of stationary phase.

Sulfolobus strain G0 regardless of MOI (Figure 6A). A positive correlation between SSV8 MOI and percent inhibition of host growth was generally observed. However, for the lowest viral stock dilution ( $\mathrm{MOI}=0.01$ ), the PI was not significantly different from uninfected control despite productive infection. The atypical host growth profile for host infected with SSV9 also persisted (Figure 6B). Amplitudes of host growth spikes change with an inverse relationship in response to MOI; however, there was no switch to the Gompertzian-like effect even at the lowest SSV9 MOI.

\section{End-Point Infection Assays Indicate SSV9 Lytic Replication}

To investigate both host and virus population dynamics in liquid culture infection assays, two large-scale trials (i.e., 1420 replicates) at higher observation frequency (every $4 \mathrm{~h}$ ) were conducted with Sulfolobus strain G $\theta$ infected with either SSV8 or SSV9 at MOI = 1 (Figure 7). Two flasks were harvested every $12 \mathrm{~h}$ from the replicates to determine virus particle counts via ESI/MS. Sulfolobus strain G $\theta$ infected with SSV8 showed typical Gompertzian-like host growth with a concomitant increase in virus particle count (Figure 7A) through $78 \mathrm{~h}$ postinfection (HPI).

SSV9-infected Sulfolobus strain G $\theta$ showed a distinct pattern from the G $\theta$-SSV8 infection. SSV9-infected growth peaked (18 HPI) followed by a rapid drop in cell density (Figure 7B). The decrease in host density was followed by an increase in SSV9 particle count (32 HPI). Interestingly, other than the initial peak in host cell density, no noticeable subsequent peaks (dampened or accentuated) were observed (Figure 7B) as in prior experiments (see Figure 5).

It was also noted that the growth inhibition did not result in an optical density value of zero. Instead, cell density stabilized at approximately one-third the average peak value. Furthermore, SSV9 titer (via ESI/MS) decreased substantially (see Figure 7B, 60-100 HPI). Although the general growth profiles were consistent with prior trials for non-lytic vs. lytic release, these latter two features were not expected (see Discussion). Together, these data indicate that SSV8 pursues canonical non-lytic virion release expected of SSVs, while SSV9 lyses host strain G $\theta$. This is also supported qualitatively by the presence of significantly more cell debris in SSV9-infected cells in small-scale infections (Figure S2).

\section{SSV9 Lytic Replication May Be Limited to Allopatric Hosts}

To determine if SSV9 lytic replication was dependent upon host strain allopatry, two isolates of Sulfolobales derived from volcanic hot springs in Kamchatka, Russia were also tested as plausible hosts for SSV9.

Liquid culture infection assays confirm that strain $\mathrm{MU}$ is resistant to infection (Figure 8A) and that strain GV is susceptible to SSV9. Post-infection titers for strain MU infection trials did not show any detectable virus; while those for strain GV showed virus particle counts on the order of $10^{9} \mathrm{VP} \mathrm{mL}^{-1}$ indicating productive infection (Figure S4). The most striking result was that strain GV growth during SSV9 infection resembles the non-lytic archetype infection dynamics observed with other SSVs (Figure 8B).

AUC analysis for MU-SSV9 trials results in no significant growth inhibition (PI $<0.05$ ), while the GV-SSV9 trial shows a significant PI $(>0.20)$ with a convincing Gompertz fit $\left(R^{2}=0.934\right)$, indicating that SSV9 exhibits a non-lytic replication profile on a sympatric host, Sulfolobus sp. strain GV (see Table S3). 


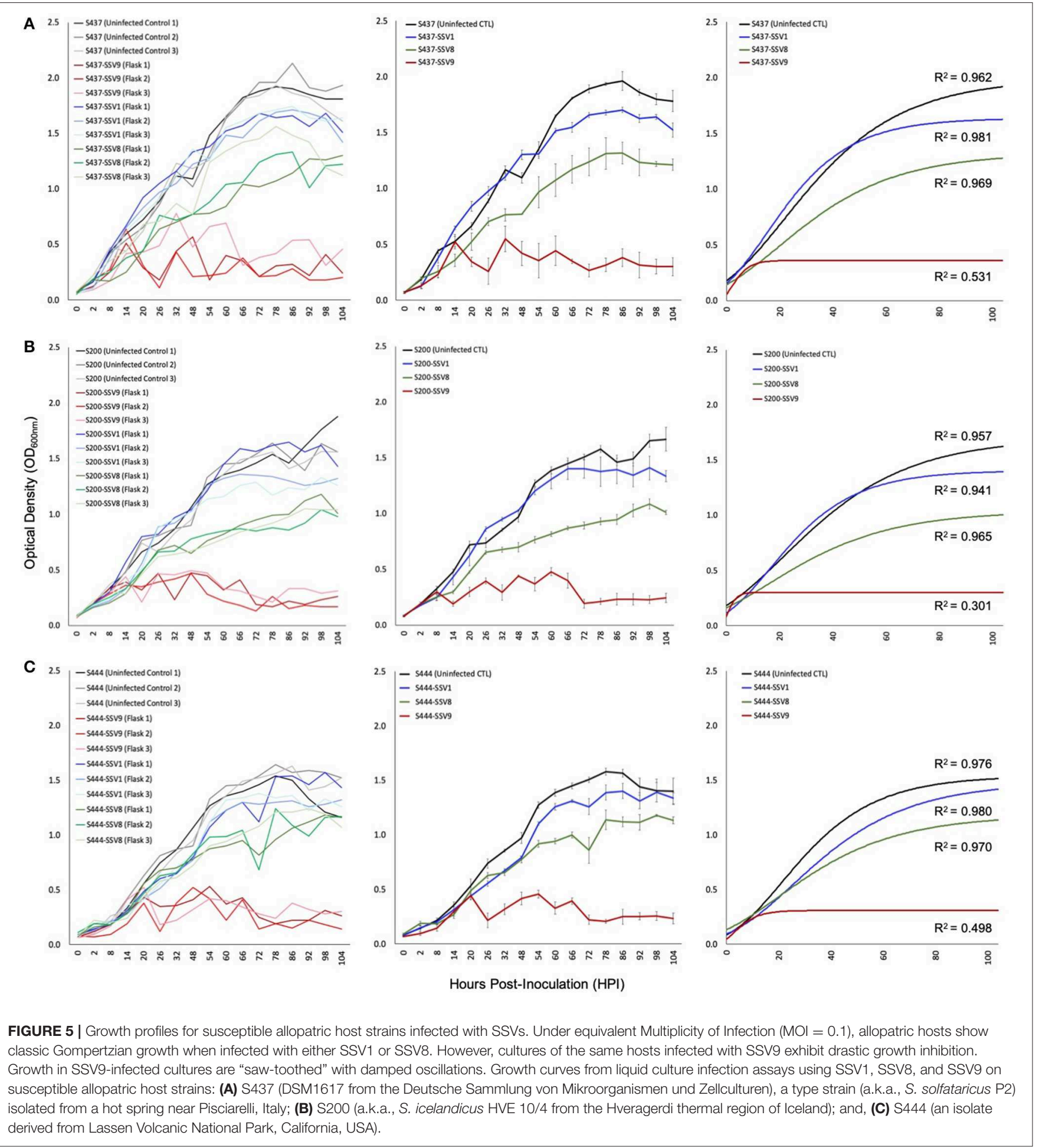

\section{DISCUSSION}

Previously published work demonstrated that some Sulfolobales are completely resistant to infection by well-characterized SSVs (i.e., SSV1, SSV2, SSV3, SSV8, SSV9, SSV10) while others are susceptible to subset or all of these SSVs in spot-on-lawn "halo" assays (Ceballos et al., 2012). Large plaque-like halos were observed on host lawns infected with SSV9 (using the same amount of virus) suggesting that SSV9 may be more virulent. However, the large clearings on host lawns generated by SSV9 appeared to be true plaques rather than the turbid halos characteristic of SSV infection. This raised the possibility that SSV9 is lysing its host rather than using non-lytic budding as a means of virion release. Although the halo assay is sufficient for determining whether a given host is susceptible (or not) to a specific SSV, it offers limited information regarding the dynamics 

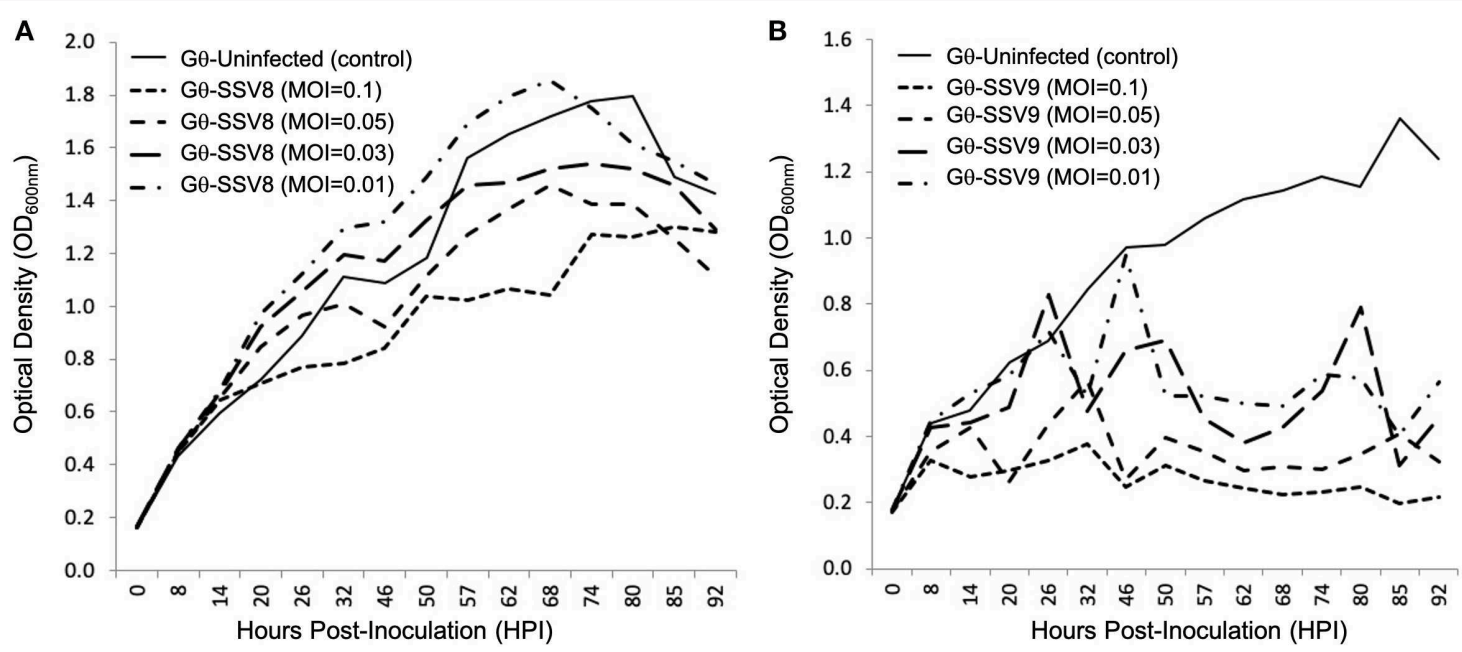

FIGURE 6 | Growth of Sulfolobus strain G $\theta$ infected at Different MOls: SSV8 versus SSV9. (A) Under different Multiplicity of Infections (0.1, 0.05, 0.03, and 0.01), strain $\mathrm{G} \theta$ infected with SSV8 retains Gompertzian growth. (B) Host strain G infected with SSV9 using the same MOls consistently results in the cyclical "saw-toothed" profile characteristic of SSV9 infection (and typical of lytic virus systems).
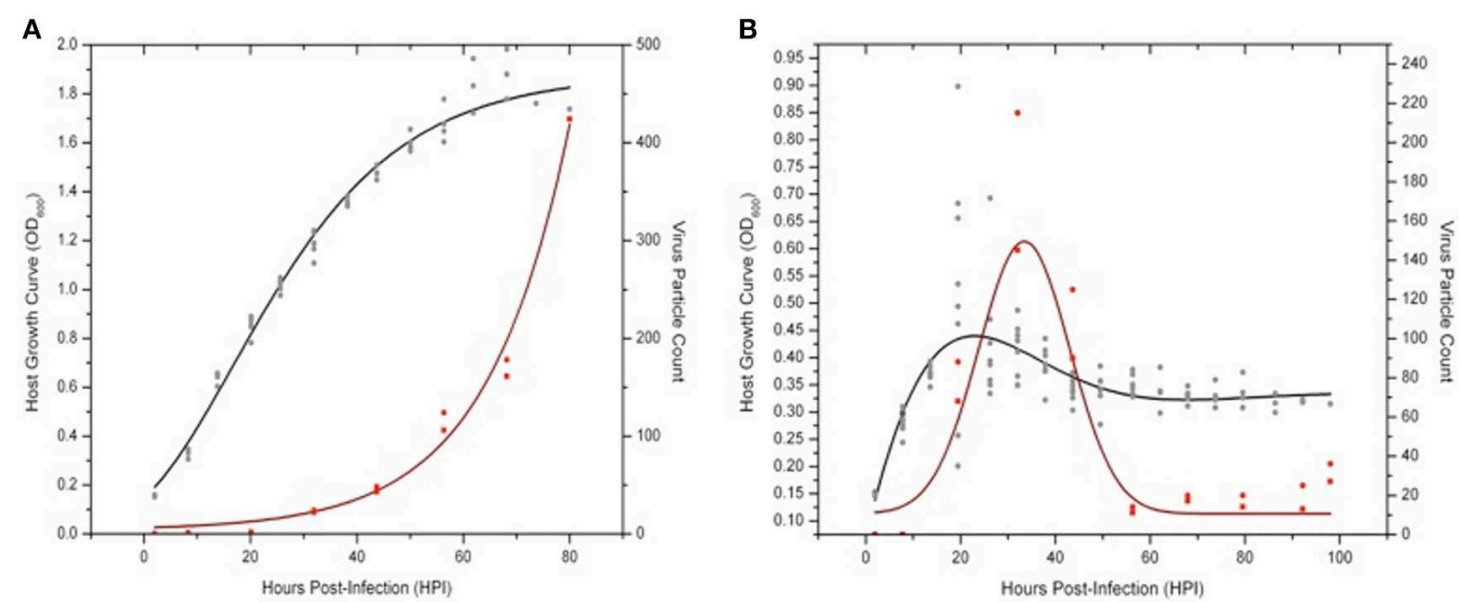

FIGURE 7 | Virus-host dynamics: SSV8 vs. SSV9 infections in Sulfolobus strain G $\theta$. Using a MOI = 1 to ensure virus particle detection via ESI/MS, host strain G $\theta$ was infected with SSV8 or SSV9. (A) Sulfolobus strain G $\theta$ at an $\mathrm{OD}_{600}=0.15-0.20$ is infected with SSV8 at MOI $=1$ resulting in standard Gomperz-like culture growth with a concomitant increase in virion count over time. (B) Sulfolobus strain $\mathrm{G} \theta$ at $\mathrm{OD}_{600} \approx 0.15-0.20$ is infected with SSV9 at $\mathrm{MOI}=1$ resulting in an average peak density of $\mathrm{OD}_{600} \approx 0.45$ followed by a decrease in cell density and a sudden increase in SSV9 particle count, peaking at $32 \mathrm{HPI}$. Cell density stabilizes at $\mathrm{OD}_{600} \approx 0.30-0.35$ from $60-90 \mathrm{HPI}$. SSV9 particle count drops to the detectable limits of the ESI/MS $\left(\sim 1 \times 10^{6}-2 \times 10^{6}\right.$ virus particles/mL)

of infection. In the present study, liquid culture assays and host growth curve analyses were used to test the hypothesis that SSV9 employs a lytic replication strategy rather than the canonical nonlytic replication (i.e., release via virus budding) characteristic of other SSVs.

\section{SSV Infection in Liquid Culture}

Growth curve analysis provides insights into the nature of virushost infection dynamics that are not resolvable through platebased halo assays. Using host growth curve analyses, it is not only possible to determine percent inhibition and relative virulence of a different SSVs on a host (see Figure 4A) but it is also possible to gain insights into rates of virus replication/transmission as well as virion release strategy. By measuring titers of inocula at the beginning of an infection trial, establishing equivalent MOI for different virus-host pairings, and taking end-point titers, a quantitative assessment of how many virus particles are present in the culture at the time of inoculation vs. how many progeny virions are present at the end-point was possible. However, this method of comparing pre-infection titer and post-infection titers may have been confounded by differential stability of distinct SSV strains in the high temperature $\left(76-80^{\circ} \mathrm{C}\right)$ and low $\mathrm{pH}(3.0-$ 3.4) culture environment. Although SSV8 and SSV9 appeared to be more resilient in solution, SSV1 did not seem to be as stable 

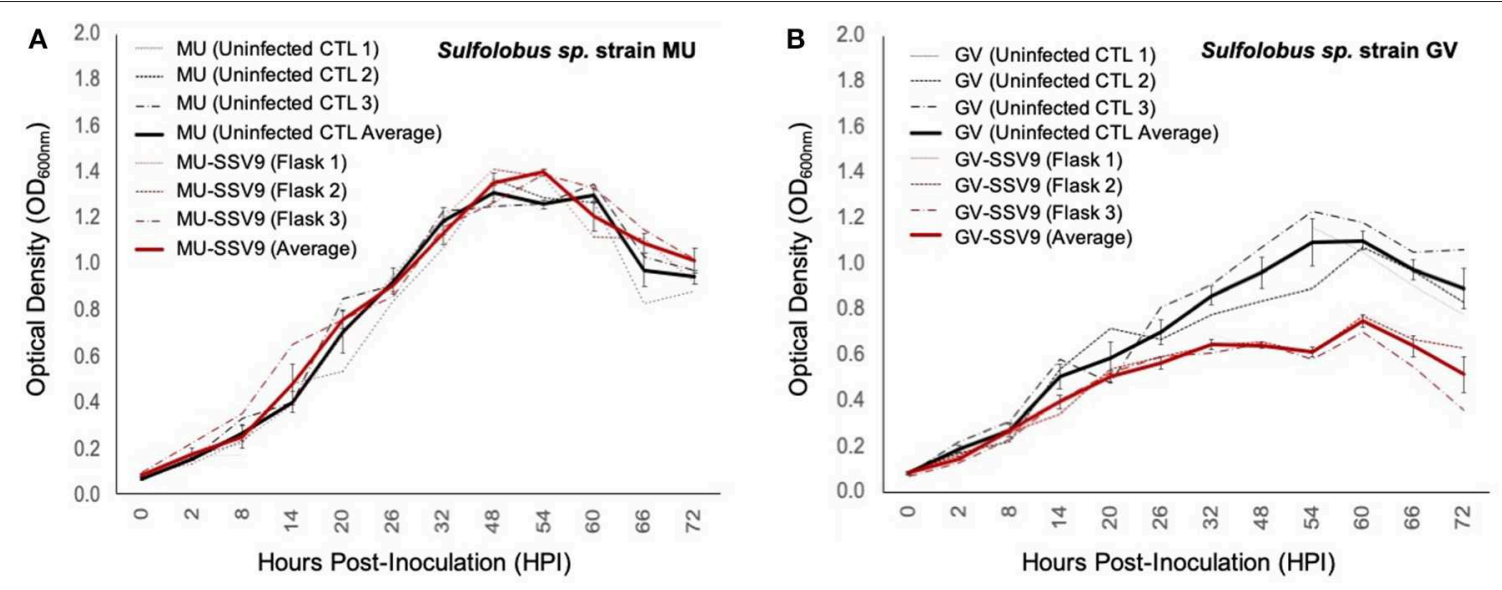

FIGURE 8 | Impacts of SSV9 challenge on isolates from geothermal regions in Kamchatka. Isolates of Sulfolobales from geothermal regions of the Kamchatka peninsula were infected with SSV9. Sulfolobus sp. strain GV was derived from the same hot spring region as the host from which SSV9 was derived. Sulfolobus sp. strain GV is considered a sympatric host. Sulfolobus sp. strain MU was also derived from the Kamchatka peninsula but from a geothermal region approximately $256 \mathrm{~km}$ to the southwest of Geyser Valley. Sulfolobus sp. strain MU is quasi-sympatric to Sulfolobus sp. strain GV. (A) Sulfolobus sp. strain MU growth curves for SSV9-infected cultures exhibit no significant changes in $\mu_{\max }$ compared to the uninfected control curves. (B) Sulfolobus sp. strain GV host growth curves for SSV9-infected cultures resemble canonical non-lytic replication.

(Figure S3). Thus, viral fecundity may have been underestimated by virus count since some virus particles (e.g., SSV1 virions) breakdown during the course of the infection assay. Still, endpoint virus count is needed to confirm productive infection. Specifically, if the number of virus particles at end-point exceeds the number of virus particles used for inoculation, then this indicates that the infection was productive. However, if virus count at end-point is equal to or less than virus particle count at inoculation, then it can be argued that no infection occurred.

Although it can be argued that a highly unstable virus strain could rapidly breakdown yielding a false negative for infection, TEM (see Figures 1, 3) and spot-on-lawn halo assays (see Figure 2) are used as secondary methods to validate host susceptibility/SSV strain infectivity.

\section{Archetypal Non-lytic SSV Replication and Host Growth Profiles}

Despite limitations in determining end-point virus count due to differences in virion stability between the different SSVs, liquid culture assays permit a quantitative assessment of relative virulence $\left(V_{R}\right)$ by calculating percent inhibition (PI) of each SSV on a given host strain (see Figure 4B). The archetypal nonlytic replication strategy of SSVs allows quantitative assessment of $V_{R}$ based on comparing $N_{\text {asymptote}}, \mu_{\max }$, and/or AUC in SSV-infected vs. uninfected controls.

\section{Atypical Infection Dynamics of SSV9 on Allopatric Host Growth}

Archetypal SSV infection (see Figure 4) induces Gompertzlike host growth profiles (Ceballos and Stacy, under review), indicative of non-lytic infection, which is consistent with turbid halo, instead of plaque, formation on spot-on-lawn plate assays. However, when SSV9 is used to infect a series of susceptible allopatric hosts, atypical infection dynamics emerge in the host growth curve. Specifically, a deep inhibition, which manifests as "saw-tooth" or "serial spike" profile in the host growth curve, occurs suggesting a different replication process (Figure 5, red traces). Although it may be suggested that SSV9 is simply more virulent on the susceptible host, this argument is refuted by the fact that neither a Gompertz or Logistics growth models fit reasonably to the SSV9-infection host growth data (as indicated by very low $\mathrm{R}^{2}$ values).

\section{Atypical Infection Dynamics of SSV9 Are Not MOI Dependent}

Further support for unique SSV9 infection dynamics rests in the inability to elicit the archetypal non-lytic growth profile by manipulating MOI (see Figure 6B). Even under low MOI, SSV9 continues to induce a cyclic spike profile in the host growth curve. Likewise, varying MOI for SSV8 does not change the archetypal non-lytic growth profile to one resembling SSV9 infection. To determine if a change in the SSV8-infect host growth profile could be forced by a concentrated inoculum, a 20:1 virus concentrate was used $(\mathrm{MOI}=2)$. Although the culture exhibited a strong depression in growth (not shown), the curve still fit a Gompertz Model with high coefficient of determination $\left(R^{2}=0.87\right)$ and did not show a cyclic growth profile. Furthermore, virus particle count at the end of a 20:1 viral concentrate trial (not shown) was 2 -fold greater than the initial inoculum, indicating that the depression in the growth curve was not due to lysis-from-without (Delbrück, 1940) but due to productive infection.

\section{SSV Population Dynamics Support Lytic Replication}

Larger-scale liquid culture assay trials (with a large number of replicate flasks) allow replicates to be harvested periodically 
during the course of infection to monitor changes in virus titer in parallel to host growth (Figure S2). Sampling at shorter intervals (i.e., every $4 \mathrm{~h}$ ), infection with SSV8 (a non-lytic replicator) shows an increase in virus particle count concomitant with host growth. However, SSV9 infection of the same host strain (i.e., G $\theta$ ) shows sharp peak in virus particle count $\sim 12 \mathrm{~h}$ after a peak and a notable rapid drop in host cell density. This is consistent with lytic virus release or a "burst" (Figure 7B). Interestingly, even though the assay continued to $\sim 98 \mathrm{HPI}$, no subsequent peak-and-crash of host (or virus) occurred after the first cycle ( $\sim 30$ HPI). Moreover, optical density (a proxy for host cell density) remained steady at $\mathrm{OD}_{600 \mathrm{~nm}} \approx 0.3$ from 60 to $98 \mathrm{HPI}$. The reason underlying the stability of host cells density with no additional SSV9-induced lysing or SSV9 virion production is not clear. There is one report suggesting that SSV9 challenge may induce dormancy in viable hosts (Bautista et al., 2015), which may be the reason for sustained absorbance at $\mathrm{OD}_{600 \mathrm{~nm}} \approx 0.3$. Indeed, dormant cells would not likely support virus production and may render cells resistant to infection.

Another report suggests that group II chaperonin complexes may form highly stable networks of chaperonin complex filaments at the intracellular surface of membrane (Trent et al., 2003). Cell membrane-associated chaperonin complexes as filaments or two-dimensional arrays can maintain cell shape even if cells are not viable. Under scanning electron microscopy (SEM), cells from infected culture, which are likely to be nonviable "ghost cells" due to the presence of large holes in the cell membrane, maintain a round lobed three-dimensional structure that could absorb $600 \mathrm{~nm}$ light (also reported in Quemin et al., 2016). Whether the absence of subsequent cycles of host recovery, SSV9 infection, and lytic bursting beyond the initial cycle are due to cells becoming dormant or whether these are "ghost" cells requires further study. Nonetheless, host growth curves for SSV9-infected strains display atypical profiles that do not fit to Gompertzian (or Logistic Growth Models) as is expected for non-lytic virion release but rather represent lytic bursts of virion release. Thus, both spot-on-lawn halo assays and liquid culture assays suggest that SSV9 lyses host. Whether this is classical lytic replication (as seen in bacteriophage) or if lysis is a result of virion activity at the membrane, which induces membrane breakdown, remains uncertain. It is possible that a "lysis-from-within" phenomena is responsible whereby aggressive virus egress is inducing gross cell lysis in allopatric hosts, which have limited coevolutionary history with SSV9; whereas the sympatric host is adapted to support typical SSV budding. Whether SSV9 infection is truly lytic replication or a "lysis-from-within" phenomenon, is uncertain. Nonetheless, small-scale infection assays further support that cells are lysed. Specifically, infection with SSV9 generates visible cell debris at the same time point of infection compared to infection with other SSVs, which do not (see Figure S2).

\section{SSV9 Lytic Replication May Depend on Host Allopatry}

Although SSV9 lytic behavior does not appear to be dependent upon MOI and seems to be an inherent property of the SSV9 replication strategy, all susceptible hosts initially tested were allopatric hosts isolated from geothermal hot springs thousands of kilometers away from where SSV9 was isolated. Yet, when two Sulfolobales-one from the same geothermal region from which the original SSV9 was isolated and another from a distant spring (ca. $250 \mathrm{~km}$ ) within the same area (i.e., Kamchatka, Russia)were challenged with SSV9, unanticipated dynamics emerged.

The quasi-sympatric strain MU was not susceptible to SSV9 in spot-on-lawn assays. Moreover, in liquid culture assays with strain MU, there were no significant differences in $N_{\text {asymptote }}, \mu_{\max }$, or AUC between the SSV9-infected and MU uninfected controls (Figure 8A). This is not surprising since other Sulfolobales are reported to be resistant to SSV infection (Ceballos et al., 2012). Yet, when SSV9 was used to challenge the sympatric strain GV, there were unexpected results. Specifically, it appears that SSV9 not only infects strain GV but infection follows canonical non-lytic replication characteristic of other SSVs (Figure 8B). It is possible that coevolution of host and virus has resulted in a host cell membrane that is more resilient to the sympatric virus assembly mechanisms at the membrane surface, while membranes of allopatric hosts are more sensitive to SSV9 assembly resulting in cell lysis.

Recently, a series of reports (Sakai and Kurosawa, 2018; Tsuboi et al., 2018) proposed a re-organization of Sulfolobaceae into four distinct genera: Sulfolobus (Brock et al., 1972), Stygiolobus (Segerer et al., 1991), Sulfurisphaera (Kurosawa et al., 1998), Saccharolobus (Sakai and Kurosawa, 2018; Tsuboi et al., 2018)_ with the latter being the most recently defined based largely on phylogenetic distance.

Whether SSV infectivity and relative virulence is correlated with these newly defined genera remains to be determined. In terms of our prior work (Ceballos et al., 2012) and this current study, this reorganization means that SSVs infect select strains belonging in at least two of these genera (i.e., Saccharolobus and Sulfurisphaera). The full impact of this taxonomic reorganization, especially with regard to biogeography and physiological differences is being assessed. Interestingly, Sulfurisphaera tokodaii (Tsuboi et al., 2018) and Sulfurisphaera ohwakuensis (Kurosawa et al., 1998) share a similar infection pattern to Sulfolobus sp. strains MU and GV. Specifically, S. tokadaii appears to be resistant to SSV infection, like strain MU; while, S. ohwakuensis is susceptible to at least a subset of SSVs tested (Ceballos et al., 2012), like strain GV. Although re-organization of the phylogenetic tree for the family Sulfolobaceae requires the community to revisit and reassess previously published work, the non-lytic growth profiles observed in SSV9 infections raises a question of whether sympatric vs. allopatric virus-host evolution or phylogenetic relatedness or both determines replication strategy. Since lytic replication typically results in greater virulence (than non-lytic replication), there is also the question of whether a switch in replication strategy over evolutionary timescales (i.e., lytic to non-lytic) is part of a more generalizable pattern of attenuation in the virus-host coevolutionary "arms race" (van Valen, 1973; Dawkins and Krebs, 1979). Furthermore, the genetic substrates underlying the emergence of distinct replication strategies in SSV systems remain unknown. Employing high throughput 
sequencing methods (e.g., nanopore sequencing) and advanced analytical techniques may resolve the impacts of specific genetic factors on virus-host dynamics and quantify relative virulence between non-lytic vs. lytic phenotypes, respectively (Ceballos and Stacy, under review), may help to address the aforementioned questions and identify genetic elements that predispose viruses to lytic vs. non-lytic replication strategy.

\section{DATA AVAILABILITY STATEMENT}

All datasets generated for this study are included in the article/Supplementary Material.

\section{AUTHOR CONTRIBUTIONS}

$\mathrm{RC}$ and $\mathrm{KS}$ conceptualized the study. RC performed the liquid culture and plate assays and did the virus titering using ESI/MS with instrumentation at BVS, Inc. (Hamilton, MT). CD performed the plate assays and conducted the SSV thermal stability and adsorption studies, and also did the virus titering using serial dilution plaque assays. CS and RC fit the data to mathematical models and determined $\mathrm{N}_{\text {asymptote, }} \mu_{\max }$, and AUC for all liquid culture assays. RC and CD produced the TEM and SEM images. EP-C performed the qPCR-based virus titers. RC prepared the initial drafts of the full manuscript and incorporated the final revisions. KS contributed to the sections of the writing and revised iterations of the manuscript.

\section{FUNDING}

Distinct components of this project were supported in part by each of the following sources: NASA EPSCoR award no. NNX07AT63A (EPSCoR PD-DesJardins; SciPI-RC) partially funded environmental sampling and strain development; U.S. NSF RIG award no. 0803199 (PI-RC) partially funded preliminary data on plate based infection assays; U.S. NSF MCB award no. 1818346 (PI-RC) partially funded liquid culture assays and some molecular analyses including the participation of CS; U.S. NSF ROA award no. 1906307 (PI-RC; coPI-EP-C) is a Research Opportunities Award supporting EP-C's engagement in the project; and U.S. NSF MCB award no. 0702020 (PI-KS) supported the work of CS and KS.

\section{ACKNOWLEDGMENTS}

The authors thank UA students Kyle Hanson and Blythe Bunkers for assisting with infection assays; David Wick (BVS,

\section{REFERENCES}

Arnhold, E. (2017). easynls: Easy Nonlinear Model. R package version. 5 (Vienna: The Comprehensive R Archive Network), 1-9.

Bautista, M. A., Zhang, C., and Whitaker R. J. (2015). Virus-induced dormancy in the archaeon Sulfolobus islandicus. MBio 6:e2565-14. doi: $10.1128 / \mathrm{mBio} .02565-14$
Inc.) for technical support with the ESI/MS tittering, and Dr. Jonathan Trent and Dr. Mark Young for providing several strains of Sulfolobales derived from different geothermal regions worldwide for comparative analyses.

\section{SUPPLEMENTARY MATERIAL}

The Supplementary Material for this article can be found online at: https://www.frontiersin.org/articles/10.3389/fmicb. 2020.01218/full\#supplementary-material

Figure S1 | SSV titers via electrospray ionization mass spectrometry (ESI/MS). Concentrated viral suspension is diluted 1:10 and mixed with ionization solution (see section Methods). SSV particle count in $120 \mathrm{~nL}$ of suspension is monitored by a particle detector for $3 \mathrm{~min}$. The area-under-the-curve (AUC) provides an estimate of titers. Each SSV has a characteristic spectrum. SSV1 (blue) is generally a single broad peak. SSV8 (green) exhibits a large single peak with a shoulder. SSV9 (red) typically presents as a double-peak. Since ESI/MS measures are based on mass:charge ratios of spherical particles and SSVs are fusiform (quasi-elliptical) shoulders and double peaks may be due to different orientations between aerosolization and triggering the detector. Alternatively, morphologically different populations may be present, including a sub-population of defective virus-like particles such as those that have lost their tails, which generate a shoulder or second peak.

Figure S2 | Cellular Debris in SSV9-infected but not in SSV1-infected Small-scaled Cultures. (A) SSV9-infected Sulfolobus strain Ge has cell debris

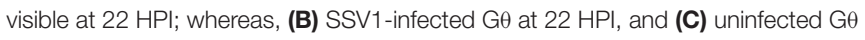
uninfected control do not. Cultures were allowed to settle overnight. Both infections were conducted at an $\mathrm{MOI}=3$. Cell debris is another line of evidence suggesting that SSV9 induces host cell lysis.

Figure S3 | SSV viability after exposure to culture environment. At $75^{\circ} \mathrm{C}(\mathrm{pH}$ 3.2) SSV9 (red) retains viability for up to $25 \mathrm{~h}$; while titers of SSV1 (blue) decrease rapidly after $6 \mathrm{~h}$ in the same high temperature acid conditions. Plate-based serial dilution plaque-like "halo" assays (hfu/mL) were used to determine titers for each time point for SSV9 and SSV1. SSV9 virus particles (red) have greater stability in the high temperature and acid environment.

Figure S4 | SSV9 genome abundance via quantitative polymerase chain reaction (qPCR). Viral genome counts were measured via qPCR after infections of Sulfolobus sp. strains MU and GV with SSV9. Liquid cultures of Sulfolobus sp. strains $\mathrm{MU}$ and GV were infected with SSV9 at an $\mathrm{MOI}=0.1$ (dark grey bars) from a SSV stock titered at $1.03 \times 10^{10} \mathrm{vg} / \mathrm{mL}$ (black bar) followed by incubation for $72 \mathrm{~h}$ at $78^{\circ} \mathrm{C}$ (90 RPM shaking). At $72 \mathrm{HPI}$, titers were determined by qPCR from culture supernatant. SSV9 genomes per $\mathrm{mL}$ in $\mathrm{MU}$ culture dropped five orders of magnitude to within the lowest detection limit (medium grey bar), which indicates no productive infection. SSV9 genomes in GV culture increased by two orders of magnitude (light grey bar), indicating that Sulfolobus sp. strain GV is permissive to SSV9 replication.

Table S1 | Percent inhibition calculations for SSV-G infection: Figure 4.

Table S2 | Percent inhibition for SSV infections on allopatric hosts: Figure 5.

Table S3 | Percent inhibition for SSV9 challenges on sympatric sulfolobales: Figure 8. 
fluorescens-Ф2 model system. Infect. Genet. Evol. 7, 547-552. doi: 10.1016/j.meegid.2007.01.005

Brumfield, S. K., Ortmann, A. C., Ruigrok, V., Suci, P., Douglas, T., and Young, M. J. (2009). Particle assembly and ultrastructural features associated with replication of the lytic archaeal virus Sulfolobus turreted icosahedral virus. J. Virol. 83, 5964-5970. doi: 10.1128/JVI.02668-08

Cannio, R., Contursi, P., Rossi, M., and Bartolucci, S. (1998). An autonomously replicating transforming vector for Sulfolobus solfataricus. J. Bacteriol. 180, 3237-3240. doi: 10.1128/JB.180.12.3237-3240.1998

Ceballos, R. M., Marceau, C. D., Marceau, J. O., Morris, S., Clore, A. J., and Stedman, K. M. (2012). Differential virus host-ranges of the Fuselloviridae of hyperthermophilic Archaea: implications for evolution in extreme environments. Front. Microbiol. 3:295. doi: 10.3389/fmicb.2012.00295

Contursi, P., Jensen, S., Aucelli, T., Rossi, M., Bartolucci, S., and She, Q. (2006). Characterization of the Sulfolobus host-SSV2 virus interaction. Extremophiles 10, 615-627. doi: 10.1007/s00792-006-0017-2

Dawkins, R., and Krebs, J. R. (1979). Arms races between and within species. Proc. R Soc. B Biol. Sci. 205, 489-511. doi: 10.1098/rspb.1979.0081

Delbrück, M. (1940). The growth of bacteriophage and lysis of the host. J. Gen. Physiol. 23, 643-660. doi: 10.1085/jgp.23.5.643

Dennehy, J. J. (2009). Bacteriophages as model organisms for virus emergence research. Trends Microbiol. 17, 450-457. doi: 10.1016/j.tim.2009.07.006

Forterre, P. (2006). Three RNA cells for ribosomal lineages and three DNA viruses to replicate their genomes: a hypothesis for the origin of cellular domain. Proc. Natl. Acad. Sci. U.S.A. 103, 3669-3674. doi: 10.1073/pnas.0510333103

Fusco, S., Liguori, R., Limauro, D., Bartolucci, S., She, Q., and Contursi, P. (2015). Transcriptome analysis of Sulfolobus solfataricus infected with two related fuselloviruses reveals novel insights into the regulation of CRISPR-Cas system. Biochimie 118, 322-332. doi: 10.1016/j.biochi.2015.04.006

Gompertz, B. (1825). XXIV. On the nature of the function expressive of the law of human mortality, and on a new mode of determining the value of life contingencies. In a letter to Francis Baily, Esq. FRS \&c. Philos. Trans. $R$ Soc. Lond. 115, 513-583. doi: 10.1098/rstl.1825.0026

Goodman, D. A., and Stedman, K. M. (2018). Comparative genetic and genomic analysis of the novel fusellovirus Sulfolobus spindle-shaped virus 10. Virus Evol. 4:vey022. doi: 10.1093/ve/vey022

Greischar, M. A., and Koskella, B. (2007). A synthesis of experimental work on parasite local adaptation. Ecol. Lett. 10, 418-434. doi: 10.1111/j.1461-0248.2007.01028.x

Grogan, D., Palm, P., and Zillig, W. (1990). Isolate B12, which harbours a virus-like element, represents a new species of the archaebacterial genus Sulfolobus, Sulfolobus shibatae, sp. nov. Arch. Microbiol. 154, 594-599. doi: 10.1007/BF00248842

Grogan, D. W. (1989). Phenotypic characterization of the archaebacterial genus Sulfolobus: comparison of five wild-type strains. J. Bacteriol. 171, 6710-6709. doi: 10.1128/JB.171.12.6710-6719.1989

Hasenbrink, G., Schwarzer, S., Kolacna, L., Ludwig, J., Sychrova, H., and Lichtenberg-Fraté, H. (2005). Analysis of the mKir2. 1 channel activity in potassium influx defective Saccharomyces cerevisiae strains determined as changes in growth characteristics. FEBS Lett. 579, 1723-1731. doi: 10.1016/j.febslet.2005.02.025

Held, N. L., and Whitaker, R. J. (2009). Viral biogeography revealed by signatures in Sulfolobus islandicus genomes. Environ. Microbiol. 11, 457-466. doi: 10.1111/j.1462-2920.2008.01784.x

International Committee on Taxonomy of Viruses (2017). Virus Taxonomy. Available online at: https://dx.doi.org/10.1093\%2Fnar\%2Fgkx932 (accessed October 13, 2017).

Iverson, E., and Stedman, K. (2012). A genetic study of SSV1, the prototypical fusellovirus. Front. Microbiol. 3:200. doi: 10.3389/fmicb.2012.00200

Kahm, M., Hasenbrink, G., Lichtenberg-Fraté, H., Ludwig, J., and Kshischo, M. (2010). grofit: fitting biological growth curves with R. J. Stat. Softw. 33:v033.i07. doi: $10.18637 /$ jss.v033.i07

Krupovic, M., Cvirkaite-Krupovic, V., Iranzo, J., Prangishvili, D., and Koonin, E. V. (2018). Viruses of archaea: structural, functional, environmental and evolutionary genomics. Virus Res. 244, 181-193. doi: 10.1016/j.virusres.2017.11.025

Kurosawa, N., Itoh, Y. H., Iwai, T., Sugai, A., Uda, I., Kimura, N., et al. (1998). Sulfurisphaera ohwakuensis gen. nov., sp. nov., a novel extremely thermophilic acidophile of the order Sulfolobales. Int. J. Syst. Evol. Microbiol. 48, 451-456 doi: 10.1099/00207713-48-2-451

Laird, A. K. (1965). Dynamics of tumour growth: comparison of growth rates and extrapolation of growth curve to one cell. Br. J. Cancer 19, 278-291. doi: 10.1038/bjc.1965.32

Lenski, R. E., and Levin, B. R. (1985). Constraints on the coevolution of bacteria and virulent phage: a model, some experiments, and predictions for natural communities. Am. Nat. 125, 585-602. doi: 10.1086/284364

Lopez, S., Prieto, M., Dijkstra, J., Dhanoa, M. S., and France, J. (2004). Statistical evaluation of mathematical models for microbial growth. Int. J. Food Microbiol. 96, 289-300. doi: 10.1016/j.ijfoodmicro.2004.03.026

Martin, A., Yeats, S., Janekovic, D., Reiter, W., Aicher, W., and Zillig, W. (1984). SAV-1, a temperate uv-inducible DNA virus-like particle from the archaebacterium Sulfolobus acidocaldarius isolate B12. EMBO J. 3, 2165-2168. doi: 10.1002/j.1460-2075.1984.tb02107.x

Moré, J. J. (1978). "The levenberg-marquardt algorithm: implementation and theory," in Numerical Analysis, ed G. A. Watson (Berlin; Heidelberg; New York, NY: Springer), 105-116. doi: 10.1007/BFb0067700

Morgan, A. D., Gandon, S., and Buckling, A. (2005). The effect of migration on local adaptation in a coevolving host-parasite system. Nature 437, 253-256. doi: $10.1038 /$ nature 03913

Munson-McGee, J. H., Peng, S., Dewerff, S., Stepanauskas, R., Whitaker, R. J., Weitz, J. S., et al. (2018). A virus or more in (nearly) every cell: ubiquitous networks of virus-host interactions in extreme environments. ISME J. 12, 1706-1714. doi: 10.1038/s41396-018-0071-7

Nadal, M., Mirambeau, G., Forterre, P., Reiter, W.-D., and Duguet, M. (1986). Positively supercoiled DNA in a virus-like particle of an archaebacterium. Nature 321, 256-258. doi: 10.1038/321256a0

Palm, P., Schleper, C., Grampp, B., Yeats, S., McWilliam, P., Reiter, W., et al. (1991). Complete nucleotide sequence of the virus SSV1 of the archaebacterium Sulfolobus shibatae. J. Virol. 185, 242-250. doi: 10.1016/0042-6822(91)90771-3

Pauly, M. D., Bautista, M. A., Black, J. A., and Whitaker, R. J. (2019). Diversified local CRISPR-Cas immunity to viruses of Sulfolobus islandicus. Phil. Trans. $R$ Soc. B. 374:20180093. doi: 10.1098/rstb.2018.0093

Prangishvili, D., Bamford, D. H., Forterre, P., Iranzo, J., Koonin, E. V., and Krupovic, M. (2017). The enigmatic archaeal virosphere. Nat. Rev. Microbiol. 15:724. doi: 10.1038/nrmicro.2017.125

Prangishvili, D., Garrett, R. A., and Koonin, E. V. (2006). Evolutionary genomics of archaeal viruses: unique viral genomes in the third domain of life. Virus Res. 117, 52-67. doi: 10.1016/j.virusres.2006.01.007

Prangishvili, D., Holz, I., Stieger, E., Nickell, S., Kristjansson, J. K., and Zillig, W. (2000). Sulfolobicins, specific proteinaceous toxins produced by strains of the extremely thermophilic archaeal genus Sulfolobus. J. Bacteriol. 182, 2985-2988. doi: 10.1128/JB.182.10.2985-2988.2000

Quemin, E. R., Chlanda, P., Sachse, M., Forterre, P., Prangishvili, D., and Krupovic, M. (2016). Eukaryotic-like virus budding in archaea. MBio. 7:143916. doi: 10.1128/mBio.01439-16

Quemin, E. R., Pietila, M. K., Oksanen, H. M., Forterre, P., Rijpstra, I. C., Schouten, S., et al. (2015). Sulfolobus Spindle-shaped Virus 1 contains glycosylated capsid proteins, a cellular chromatin protein, and host-derived lipids. J. Virol. 89, 11681-11691. doi: 10.1128/JVI.02270-15

Rajnovic, D., Muñoz-Berbel, X., and Mas, J. (2019). Fast phage detection and quantification: an optical density-based approach. PLOS ONE 14:0216292. doi: 10.1371/journal.pone.0216292

Reiter, W., Palm, P., Yeats, S., and Zillig, W. (1987). Gene expression in archaebacteria: physical mapping of constitutive and UV-inducible transcripts from the Sulfolobus virus-like particle SSV1. MGG 209, 270-275. doi: 10.1007/BF00329653

Reiter, W.-D., Palm, P., and Yeats, S. (1989). Transfer RNA genes frequently serve as integration sites for prokaryotic genetic elements. Nucleic Acids Res. 17, 1907-1914. doi: 10.1093/nar/17.5.1907

Reno, M. L., Held, N. L., Fields, C. J., Burke, P. V., and Whitaker, R. J. (2009). Biogeography of the Sulfolobus islandicus pan-genome. Proc. Natl. Acad. Sci. U.S.A. 106, 8605-8610. doi: 10.1073/pnas.0808945106

Sakai, H. D., and Kurosawa, N. (2018). Saccharolobus caldissimus gen. nov., sp. nov., a facultatively anaerobic iron-reducing hyperthermophilic archaeon isolated from an acidic terrestrial hot spring, and reclassification of Sulfolobus solfataricus as Saccharolobus solfataricus comb. nov. and Sulfolobus shibatae 
as Saccharolobus shibatae comb. Int. J. Syst. Evol. Microbiol. 68, 1271-1278. doi: 10.1099/ijsem.0.002665

Schleper, C., Kubo, K., and Zillig, W. (1992). The particle SSV1 from the extremely thermophilic archaeon Sulfolobus is a virus: demonstration of infectivity and of transfection with viral DNA. Proc. Natl. Acad. Sci. U.S.A. 89, 7645-7649. doi: $10.1073 /$ pnas.89.16.7645

Segerer, A. H., Trincone, A., Gahrtz, M., and Stetter, K. O. (1991). Stygiolobus azoricus gen. nov., sp. nov. represents a novel genus of anaerobic, extremely thermoacidophilic archaebacteria of the order Sulfolobales. Int. J. Syst. Evol. Microbiol. 41, 495-501. doi: 10.1099/00207713-41-4-495

Sprouffske, K., and Wagner, A. (2016). Growthcurver: an R package for obtaining interpretable metrics from microbial growth curves. BMC Bioinform. 17:172. doi: 10.1186/s12859-016-1016-7

Spurr, A. R. (1969). A low-viscosity epoxy resin embedding medium for electron microscopy. J. Ultrastruct. Res. 26, 31-43. doi: 10.1016/S0022-5320(69)90033-1

Stedman, K. M. (2008). Fuselloviruses of Archaea. New York, NY: Academic Press.

Stedman, K. M., She, Q., Phan, H., Arnold, H. P., Holz, I., Garrett, R. A., et al. (2003). Relationships between fuselloviruses infecting the extremely thermophilic archaeon Sulfolobus: SSV1 and SSV2. Res. Microbiol. 154, 295-302. doi: 10.1016/S0923-2508(03)00074-3

Trent, J. D., Kagawa, H. K., Paavola, C. D., McMillan, R. A., Howard, J., Jahnke, L., et al. (2003). Intracellular localization of a group II chaperonin indicates a membrane-related function. Proc. Natl. Acad. Sci. U.S.A. 100, 15589-15594. doi: 10.1073/pnas.2136795100

Tsuboi, K., Sakai, H. D., Nur, N., Stedman, K. M., Kurosawa, N., and Suwanto, A. (2018). Sulfurisphaera javensis sp. nov., a hyperthermophilic and acidophilic archaeon isolated from Indonesian hot spring, and reclassification of Sulfolobus tokodaii Suzuki et al. 2002 as Sulfurisphaera tokodaii comb. Int. J. Syst. Evol. Microbiol. 68, 1907-1913. doi: 10.1099/ijsem.0.002765

van Valen, L. (1973). Body size and numbers of plants and animals. Evolution 27, 27-35. doi: 10.1111/j.1558-5646.1973.tb05914.x

Whitaker, R. J., Grogan, D. W., and Taylor, J. W. (2003). Geographic barriers isolate endemic populations of hyperthermophilic archaea. Science 301, 976-978. doi: 10.1126/science.1086909

Wick, C., and McCubbin, P. (1999). Characterization of purified MS2 bacteriophage by the physicalcounting methodology used in the integrated virus detection system (IVDS). Toxicol. Mech. Method. 9, 245-252. doi: 10.1080/105172399242591

Wick, C. H., Jabbour, R. E., McCubbin, P. E., and Deshpande, S. V. (2006). Detecting bacteria by direct counting of structural protein units by IVDS and mass spectrometry. Toxicol. Mech. Method. 16, 485-493. doi: 10.1080/15376510600910477

Wiedenheft, B., Stedman, K., Roberto, F., Willits, D., Gleske, A., Zoeller, L., et al. (2004). Comparative genomic analysis of hyperthermophilic archaeal Fuselloviridae viruses. J. Virol. 78, 1954-1961. doi: 10.1128/JVI.78.4.1954-1961.2004

Zhang, J., Zheng, X., Wang, H., Jiang, H., Dong, H., and Huang, L. (2020). Novel Sulfolobus fuselloviruses with extensive genomic variations. J. Virol. 94, e01624-e01619. doi: 10.1128/JVI.00192-20

Zillig, W., Arnold, H. P., Holz, I., Prangishvili, D., Schweier, A., Stedman, K. M., et al. (1998). Genetic elements in the extremely thermophilic archaeon Sulfolobus. Extremophiles 2, 131-140. doi: 10.1007/s007920 050052

Zillig, W., Prangishvili, D., Schleper, C., Elferink, M., Holz, I., Albers, S., et al. (1996). Viruses, plasmids and other genetic elements of thermophilic and hyperthermophilic archaea. FEMS Microbiol. Rev. 18, 225-236. doi: 10.1111/j.1574-6976.1996.tb00239.x

Zwietering, M. H., Jongenburger, I., Rombouts, F. M., and Van't, Riet, K. (1990). Modeling of the bacterial growth curve. Appl. Environ. Microbiol. 56, 1875-1881. doi: 10.1128/AEM.56.6.1875-1881.1990

Conflict of Interest: The authors declare that the research was conducted in the absence of any commercial or financial relationships that could be construed as a potential conflict of interest.

Copyright (C) 2020 Ceballos, Drummond, Stacy, Padilla-Crespo and Stedman. This is an open-access article distributed under the terms of the Creative Commons Attribution License (CC BY). The use, distribution or reproduction in other forums is permitted, provided the original author(s) and the copyright owner(s) are credited and that the original publication in this journal is cited, in accordance with accepted academic practice. No use, distribution or reproduction is permitted which does not comply with these terms. 